\title{
CONNOTATIVE FACETS OF MEANING IN TRANSLATION WITHIN INCONGURENT CONTEXTS
}

\author{
Dalibor Kesić, Emir Muhić \\ Faculty of Philology, University of Banja Luka, Bosnia and Hercegovina \\ E/mail: dalibor.kesic@flf.unibl.org
}

\begin{abstract}
Meanings can sometimes have unclear roots and different paths of genesis. They take us into unexplored and uncharted waters of primordial experience. Metaphoric and transferred meanings are just the mere linguistic surface of symbols. Symbols on the other hand owe their prowess to the fact that they link the semantic content with the pre-semantic depths of human experience and two-dimensionality of their structure. Lack of transparency of symbols combined with the strife to translate them exactly seems to pose an unsolvable problem which lies in the fact that all transferred meanings are indeed deeply rooted in the realm of our individual and collective experience. The perplexity of individual versus collective experiencing of particular meanings is further confounded in the cases of meaning transferability and translatability.
\end{abstract}

Key words: translation, metaphor, transferred meaning, effects, principles

\section{BACKROUND DEVELOPMENT}

Accidentally or not, some segments of semantics remain marooned and cohuned outside of the reach of mainstream linguistics. That is particularly the case with the crosssection between semantics and semiotics, more specifically, with the case of transference of symbols from one domain into another; from the actual prime meanings into their speculative secondary derivations. A transferred meaning is there to emanate reality in a somewhat roundabout way. In order to crack the nutshell of meaning and take a peek into its core one has to arm themselves with achievements of the decades of painstaking and fecund work of linguistic artisans of the present and the past. Furthermore so as the great achievements in general linguistics have yielded great achievements in translation studies, which is particularly true in the case of the development of structural linguistics, the work of Ferdinand de Saussure and the Moscow and Prague linguists, as well as of Chomsky and other transformational linguists who have made portentous breakthroughs in comprehending and describing the processes of semantic transformations, which, in turn, sketched the trajectory of the subsequent development of translation studies.

Comparisons between the real world and a metaworld, or illustrations that depict our existence with a touch of artistic ingenuity seem to be ubiquitous in all cultures of the world. Transference of meaning based on metaphorical comparison existing in one language need not necessarily exist in another. Such an observation of divergent linguistic capacities among the various languages on the globe still comes short of providing us with a recipe how to approach translation of metaphorical meanings with any warranty that the end 
product will be endowed with the same amount of expressiveness as whence we initially ventured to transplant it into another language and another culture. Eugen Nida described it as a dialectical voyage of text from the source language, via analysis, and then transfer, up to restructuring and, eventually, translation in the target language (1969).

\section{CUltural RoOTs AND PoststruCTURALIST APPROACH}

At a rudimentary scientific level, semantic and translation pundits can parse texts and pontificate what is a sound transference of meaning and which translation renderings thereof are good and which are bad. Even more than that, they are concerned with the different options that translators can utilize and the ways they can be adapted in conformity with the historical, sociological and cultural context. Peter France points out to the fact that theoreticians today have a far more complex task that the mere postulation of principles (2000). While, in the past, the preponderance of scientific research was placed on the comparison of the original with the product of translation, often with a latent aim to discover what has been 'lost' in translation, in the current, poststructuralist approach there seems to be a dramatic turn so that the ultimate aim is no longer to evaluate but rather to understand what has happened during the process of translation of metaphors and transferred meanings from one literary system into another. That, however draws on Peter Newmark and his instrumentality within translation theory as he purported that "Translation theory's main concern is to determine appropriate translation methods...it provides a framework of principles, restricted rules and hints for translating texts and criticizing translations..."(Newmark, 1982, p.19).

One could even argue that metaphorical expression is free from the bonds of the common understanding of the world. It might be the very reason why it is endowed with the capacity to create and introduce innovation in language. At a cursory glance, it seems that globalization has also had its antithesis epitomised in the increased interest for one's one cultural roots and one's own inherent identity which is apparent in the proliferation of scientific subjects and courses that go beyond linguistic technicalities and aim to elucidate the wider anthropological base.

On top of that, translation theory has gone in such a multitude of directions during the past two decades that there is hardly any consensus on the fundamental principles of translation of common, literal meaning, so much so that to translate a transferred meaning poses a major challenge to the translator bearing in mind the enormous differences between cultural and linguistic approaches. Thus, the translator, as the Irish theoretician Michael Cronin nicely describes it, is also a passenger, one who roams from one culture to another (2000).

Modern linguistics and its proponents from Max Blanc to George Lakoff also compels us to appreciate personal experience as an untranslatable aspect of an utterance (, notwithstanding the fact that it still includes the whole complexity of human language, further bamboozled by fact that some of those utterances have an utterly subjective nature leading us to the conclusion that any transference of meaning attempted in a translated text is a precarious endeavour whose satisfactory outcome should never be taken at face value.

That means that if a translator stumbles upon clear elements of poetic creativity, which typically includes transference of meaning, he or she actually needs to be guided by the criteria that surpass mere linguistics, as translation now includes the process of decoding and recoding. If that is the starting point from which the translator ventures into 
greater depths of the analysis of meaning, then one can expect that the subsequent translation quest will engender other, far more complex problems. A frequently encountered strategy to offset the shortcomings transferred meaning in translation is to resort to assimilation. Assimilation facilitates the matching and compatibility of images that hitherto might have been deemed untranslatable.

Superimposing one semantic filed on top of another is limitless and opens door to infinity of interpretations. Namely, the modern day trend of fast travel around the globe has its ramifications on the translation process. Consequently, translation is not seen as a sheer transposition of texts from one language into another. It can also be seen as a process of negotiation between texts and cultures, a process involving a number of transformations, all conducted via the agency of the translator. From this, it transpires that we cannot claim that notions expressed in any two languages are ever fully equivalent since each of their units entails a set of untranslatable associations and connotations. In other words, metaphorical analysis begins where the lexical coding ends.

The ancient rhetoricians would reply that the purpose of such figures of speech is either to fill in certain semantic gaps in the lexical coding, or to embellish someone's discourse and make it more palatable. Thus, the central consideration is placed on the figurative meanings of words, those meanings that are only functional when combined in larger strings. Another issue however may appear here - how does one choose which meaning is primary and why are there such deviations in meaning to begin with?

Taking into consideration that we all have more ideas than words to express them, we must stretch the meanings of those that we have at hand, sometimes beyond the realm of their everyday use. And yet, there are cases in which the proper word to denote a certain notion already exists, while, at the same time, a word that involves a transference of meaning is still the chosen one - particularly when authors wish to convey subconscious messages. This second strategy of using a transferred meaning reflects one of the key aspects of the general purpose of rhetoric or persuasion.

\section{INSTABILITY OF MEANING DELIENATION}

Every sign and every phrase likewise is a translation of another sign and another phrase (Paz; 1992). That argument however, can also be viewed from another angle without any challenge to its veracity - namely, all texts are original since every translation is distinct. Even the source meanings attempted to be translated can hardly be described as stable. Every text is unique and is at the same time a translation of another text since no text is inherently original because language itself is a particular sort of translation: primarily a translation of the nonverbal world around us. It still does not mean that a transferred meaning cannot be paraphrased if there is a steady expression in the target language with an identical meaning.

Such a paraphrasing however should not be delineated with boundaries existing in the original metaphor, bearing in mind the fact that paraphrasing can never generate the same amount of innovation as the original one. Perhaps, it would be true to say that only those transferred meanings which include an individual reference, a tertiary meaning, cannot be translated - particularly if the link with the denoted object has been lost and if they actually only exist in language owing to the secondary - transferred meaning. Due to the fact that full equivalence (in terms of synonymy or sameness) cannot be found in any of 
his categories, Jacobson states that poetic art is consequently technically untranslatable, and that rather than translation, there can only be creative transposition; whether as an intralingual transposition - from one poetic form into another, or as an intrasemiotic transposition - from one system of signs into another, that is, from the verbal into the musical, dancing, theatrical or graphical systems (1959). If this fragmentation of meaning into primary, secondary and tertiary - sounds complicated, perhaps the invoking of Roman Jakobson's famous fragmentation of translation could shed some light on the issue.

In his paper „On Linguistic Aspects of Translation“ he differentiates between three types of translation (1959): (i) Intralingual translation, or the use of other words (interpretation of verbal signs via the faculty of other signs of the same language); (ii) Interlingual translation (interpretation of verbal signs via the faculty of verbal signs of another language); (iii) Intersemiotic translation or transmutation (interpretation of verbal signs of a specific language with nonverbal signs of another sign system). Once the structure of the approaches is laid down in such a tripartite alliance, the inquiry into the most adequate one will depend entirely on its purpose.

The tripod system yields a tripod choice. It transpires then that even though expressions are in fact adequate interpretations of the units of a system, very often in translation there is no full equivalence of the ratios between the three systems. Even obvious synonyms do not yield full equivalence, particularly since, as Jacobson puts it, interlingual translation frequently has to resort to a combination of code units to fully interpret the meaning of the unit being translated, since, at different levels, different players come into equation (1959).

That revision, to put it in simple words, shifts the problem of transferred meaning from the semantics of words onto the semantics of sentences. Analysis of transferred meanings goes back as far as ancient rhetoricians but such an analysis does not fulfil the role we would expect from it, unless an important revision is made. Take for example the grammatical incongruence existing in different languages that can lead to all kinds of misunderstandings. Such incongruence can also result in a more profound semantic parting between counterpart versions of the same expression.

Perhaps an even better illustration of that point can be found in comparisons made by Benjamin Whorf (which may not be so relevant for this topic, but are nevertheless listed as a quintessential theoretical premise) between a 'temporal language' such as 'English' and a 'tenseless language' such as Hopi (Whorf, 1956). That, however, is not always the case with the meanings of utterances, particularly if they contain a transferred meaning pertaining to a culture. The vagueness of meaning and efforts to translate it accurately are related to the fact that all transferred meanings are indeed rooted in our experience which can be explored from different angles - at a certain level, they are common and communal, while at another they may be utterly individual.

The clash of inherent views on the world inculcated in linguistic expressions is particularly relevant for the modern era when the political, geographical and cultural boundaries are more permeable and less of an impediment that ever since the movement of people belonging to different linguistic realms is taking place at an unprecedented rate. That, however, is not always the case with the meanings of utterances, particularly if they contain a transferred meaning belonging to a culture and it might be worthwhile to reflect on the terminology of the so called negative turn which is used to elaborate and explain the deficiencies and shortcomings of segmented translation in comparison to those methods that observe and acknowledge the in-depth structure of metaphorical meaning. 
Namely, if one undertakes a meticulous segregation and fragmentation of expressions, notwithstanding larger semantic units and without due respect to cultural aspects of the given text, any of the following issues can ensue - a) information can be misconstrued (as is the case with different kinds of ambiguity so prevalent in the modern day); b) the original text may not be sufficiently fathomed (this is particularly conspicuous in technical and scientific translation); or c) the internal relations within the expression may be lost altogether (cultural and semantic facets). Invoking several scientific disciplines which could hopefully set the scene for new ideas regarding translation as a means of changing and innovating the history of literary creation inspired Gideon Toury to reexamine the interdisciplinary and interdicurisivity approach, placing a far greater emphasis on the target language. His inspiration laid foundations for the subsequent systemic elaboration of the subject embodied in the nascent fields of translation theory and translation studies.

On top of that, the translation theory has gone in such a multitude of directions during the past two decades that there is hardly any consensus on the fundamental principles of translation of common, literal meaning, so much so that to translate a transferred meaning poses a major challenge to the translator bearing in mind the enormous differences between cultural and linguistic approaches.

Consequenstly, those who hope to find clues for a proper translation of transferred meanings in formal translation studies may be a wee bit disappointed since it has a tendency to become a norm setting manual, and a rulebook for a translation guild movement - it would appear that translation policy is yet to deliver a golden standard Mona Baker sought equivalence at the rank level while Peter Newmark's paradigm explored it from the point of view of naturalness and directionality. That may additionally complicate the prescribed standards that translators are trained to abide by.

Having put it that way, it is only fair to say that the current globalization has also had its antithesis inculcated in the increased interest for one's own cultural roots and identities. This does not mean that there is no way to translate transferred metaphorical meanings. They may be paraphrased if there is a solid and frequently used form with an identical meaning - notwithstanding the lexical composition - for as long as such paraphrasing is not wrought with uncomplimentary effects existing in either of the two cultures or language domains - some fixed expressions make use of body parts and terms which may be tabooed in another language and culture.

When opting for such an approach to translating a transferred meaning the translator must also be aware of the possible loss of emotional properties that the original metaphor had. Numerous authors have expressed their opinions about the relationship between the creation of metaphorical meaning and the translational modelling. That affinity plays a decisive role in the papers of Max Black, one of which is even titled Models and Metaphors. That book prompted Ian Ramsey, an English theology scholar, to attempt to explain the function of religious language revising the theories of Max Black in his book Models and Mystery (1964). Transferred meanings have the ability to establish a conceptual diversity, an infinite number of potential interpretations at the conceptual level. 


\section{THE NECESSITY OF PRAGMATISM}

It does not really matter whether one opts for the first or the second approach listed at the end of the previous chapter, they have one thing in common - they both tread the thin line between models and transferred meanings enabling us to facilitate the translation by relying on referential dimensioning. True translation work is always pragmatic, and the translator sifts through a variety of different options hoping to find the one that yields a maximum of meaning compatibility and a minimum of semantic deviation - an intuitive sprout of a minimax strategy. Furthermore so since, as Octavio Paz used to say, every translation is an invention in a way, and, as such, it automatically yields a unique text (1992). If we believe that a culture of nation is dynamic, then the terminology used to describe its social determinants must also be dynamic and prone to changes. Will those changes go simultaneously in different countries? It is not easy to provide an answer to such a question. However, what we are able to observe in the world around us tells us that it is not really so. Therefore, instead of talking about incorrect and inconsistent translations we should rather be pragmatic and talk about translations in incompatible periods of time.

In such a paradigm, being pragmatic is no longer a matter of choice, but rather a necessity, particularly if we bear in mind that the cutting edge of translation is also the pillar of a culture (as Bhabhae described it metaphorically) (Bhabhae, 1994). From an extreme point of view, one can even argue that the essence of the transference of meaning lies within the decomposition of the world as we know it. In such a postcolonial view, every translation ought to start from the contemplation of the purpose it is meant to serve in the target language, or at least that is what the translation skopos urges us to do.

The relations between the source and target text and the inequality of their status needs to be observed from rational angels. Nowadays, both the original and the translation are seen as equal products of creativity of both the author and the translator, even though, as Paz observed it, their tasks are different. The author's task is to assemble words in an ideal, unalterable manner, while the translator's task is to set those words free from the shackles of the source text and invigorate them to live a new life in the target language (1992). Such synergy between the semantic polymorphism and connotative association is inherent in expressions with a transferred meaning and that is what sets them apart from plain language expressions.

If no concept can fully circumscribe a certain phraseological semantic field, then it simply means that no categorization can encompass all semantic possibilities of the given phraseological unit. What goes hand in hand with connotation is expressiveness, which is an essential ingredient in the cuisine of every great orator. Likewise, the functioning of similarities, which is so typical of symbols, can be correlated with the corresponding process in metaphors.

The relation between similarities and differences in metaphorical translations is therefore actually a conflict between different categorizations of reality, one that existed and the other one that is being born. It has often been said that transferred meanings cannot be translated - some have even postulated that the main trait of metaphor is its untranslatability because different cultures conceptualize the world in different ways and metaphors are characterized as being culture-specific which leads to conclusion that there is no simplistic general rule for the translation of metaphor, but the translatability of any given SL metaphor depends on (1) the particular cultural experiences and semantic associations exploited by it, and (2) the extent to which these can, or cannot, be reproduced non-anomalously into the TL, depending on the degree of overlap in each particular case (Dagut, 1976: 32) - which is actually defied in the translation practices. 
Understandably, such practice varies and in some cases could actually point out to the opposite as a certain percentage of metaphoric expressions actually make it easy to translate on account of their common composition in both the target and the source language (Nida, 1969: 122). One thing is for sure, the introduction of interdisciplinarity in translation can greatly facilitate transposition of transferred meanings from one language into another, or at least, it can help us deal with it with creativity rather than defeatism.

\section{CONCLUSION}

If transferred meanings are indeed to be viewed through the prism of their referential properties, then it would appear that what they have in common with the language of exact sciences is the notion that objectivity and reality can only be reached through digressions whose aim is to penetrate into our common visions and the language that we ordinarily use to describe them. Even though the function of translation in the target language is not the only criterion used for translation evaluation it is still a credible benchmark to be observed and to claim that there is a perfect translation would be equal to claiming that there is a perfect poem, a perfect novel or any other text or exclamation for that matter.

Just as the quality of a novel or a poem is illusive and unfathomable, the transference of meaning in metaphorical construction likewise remains an entity that exists only in the minds of those who are aware of their polysemy. The themes expressed in them and the domains they stem from are so diverse and adaptable in meaning that they can cover almost any aspect of the world around us. They are archetypal vehicles of transmitting thoughts. They bridge gaps pared by formality with astuteness and razor sharp precision. Their existence is ubiquitous and unchallenged in all the languages of the world. More often than not, transferred meanings are culture-bound and language restricted. In other words, transference of meaning based on metaphorical comparison existing in one language need not necessarily exist in another.

It is the incongruence among the languages and cultural contexts that causes instability and sometimes inequality in attempts to translate them with any reasonable amount of concurrence. If we were to imagine the inventories of metaphors and phraseological units as an arsenal of tools available in different quantities in different languages, then the counting of troops would tell us that the outcomes of the semantic battles are anything but certain. And yet, such an observation of divergent linguistic capacities and properties among the various languages on the globe comes short of providing us with a recipe how to approach translation of metaphorical meanings with any warranty that the end product will be endowed with the same quantity and quality of expressiveness as whence we initially ventured to transplant it in another language and another culture, particularly since such mimicry can only exist at a mythological level of discourse and it is there to emanate reality in a somewhat roundabout way.

Throughout such processes, transferred meaning expressions as well as the scientific metalanguage relate to a sort of reality that is even more real that the real world around us. As a consequence of that, the ancient mantra that the translator must be faithful to the original begins to dissipate. Transferred meaning expressions do not precisely tell us about the actual world that surrounds us - instead, they tell us what it is like.

Such comparisons between the real world and a metaworld, or illustrations that depict our existence with a touch of artistic ingenuity seem to be ubiquitous in all cultures of the 
world. However, the apparent inability to make an airtight match between a metaphorical expression in one language with that in another actually postulates the principle that there can be no absolute equivalence between any two languages and one can only concede that there are losses and gains in translation that we all have to live with.

\section{REFERENCES}

Bhabha, H (1994) The Location of Culture. London and New York: Routledge.

Black, M. (1962) Models and Metaphors. New York: Cornell University Press.

Cronin, M (2000) Across the Lines: travel, language, translation. Cork: Cork University Press.

Dagut, M. (1976). Can metaphor be translated? International Journal of Translation, XXII (1), 21-33. Brussels: Babel.

France, P (2000) Translation Studies and Translation Criticism, in Peter France ed. The Oxford Guide to Literature in English Translation. Oxford: Oxford University Press.

Jakobson, R (1959) „On Lingusitic Aspects of Translation“, in R.A. Brower (ed), On Translation. Cambridge, Mass.: Harvard University Press.

Newmark, P. (1982). Approaches to Translation. Oxford: Pergamon Press Ltd.

Nida, E, i Taber, C. (1969) The Theory and Practice of Translation. Leiden: E. J. Brill.

Paz, O. (1992) Translation: Literature and Letters, transl. Irene del Corral, in Rainer Schulte and John Biguenet eds. Theories of Translation. An Anthology of Essays from Dryden to Derrida. Chicago: University of Chicago Press.

Ramsey, I. (1964) Models and Mystery. New York: New York University Press.

Whorf, B. (1956) Science and Linguistics, New York: Caroll 